\title{
The influence of dexamethasone treatment and successive road transport stress on the occurrence of caprine pneumonia in a hot humid tropical environment
}

\author{
Benjamin Obukowho Emikpe, Polycarp N Tanko, Opeyemi Mayowa Onilude and Mohd Yussof Sabri \\ Department of Veterinary Pathology \& Microbiology, \\ Faculty of Veterinary Medicine, Universiti Putra Malaysia, 43400 UPM Serdang, Selangor, Malaysia. \\ Corresponding author: Mohd Yussof Sabri, email: sabri@vet.upm.edu.my \\ Received: 21-11-2012, Revised: 21-01-2013, Accepted: 25-01-2013, Published online: 16-05-2013
}

How to cite this article: Emikpe BO, Tanko PN, Onilude OM and Sabri MY (2013) The influence of dexamethasone treatment and successive road transport stress on the occurrence of caprine pneumonia in a hot humid tropical environment, Vet World 6(8): 497-501, doi: 10.5455/vetworld.2013.497-501

\begin{abstract}
Aim: The information on the effect of multiple stress and caprine pneumonia especially in a hot humid environment is limited in literature.

Materials and Methods: Sixteen goats were divided into 4 groups. Group A were subjected to 8 hours and 3 hours transportation, group B to 8 hours transport stress once and dexamethasone injection, group C to 8 hours transport only while group D was the control. All goats were observed for respiratory signs while temperature was monitored daily. Dead ones were necropsied while the survivors and the goats in group D were sacrificed on day 21. The clinical, gross, histopathological, immunohistochemical scores were according to standard methods.

Results: The mean total clinical score was higher in group B than group A, C and D. Two goats of the groups A, B and C goats died 21, 14 and 7 days post treatment. The dead goats in groups A, B, C had lung lesions typical of pneumonic pasteurellosis. An average of the lung consolidation of dead animals in group A was 15\%, in group B, 8.5\% and group C, $6.5 \%$ mostly involving the anterior and ventral part of the lung. The immunostaining results was also similar with all the lung samples positive for both Pasteurella multocida and Mannheimia haemolytica especially in the groups A, B, C with enhanced severity in $\mathrm{A}>\mathrm{B}>\mathrm{C}$.
\end{abstract}

Conclusion: This further buttress the need to reduce stress in farm animals and the emergence of $P$. multocida over $M$. haemolytica in the epidemiology of bacterial caprine pneumonia in stressed goats in Malaysia.

Keywords: caprine pneumonia, dexamethasone treatment, humid tropical environment, road transport stress

\section{Introduction}

Pneumonia is one of the most common respiratory problem in small ruminants throughout the world with its attending increased cost of treatment. The most important bacterial causes of caprine pneumonia are Pasteurella multocida and Mannheimia haemolytica. Often, these pathogens occur as a result of poor management practices or as a consequence of severe stress. Stressful conditions including transportation, viral infections, overcrowded pens, poor housing conditions, sudden environmental changes, had been reported to increase goats' susceptibility to these bacterial pneumonias [1].

Observable clinical signs of acute respiratory distress usually develop within 10 to 14 days in adult animals after being exposed to stress. The role of stress either alone or in combination with other management lapses in the natural incidence of pneumonic pasteurellosis had been well documented especially that of distant transport or shipping in cattle [2], sheep [3] and goats $[4,5]$. Artificially induced stress by administration of certain drugs and the susceptibility to pneumonia had also been reported to be similar to natural incidence [1].

This article is an open access article licensed under the terms of the Creative Commons Attribution License (http://creativecommons. org/licenses/by/2.0) which permits unrestricted use, distribution and reproduction in any medium, provided the work is properly cited.
Goats in the tropics are often reared under the traditional extensive management system and are transported over a considerable distance for the livestock to be marketed or slaughtered outside where they are being produced [6]. This transportation, an age long inevitable husbandry practice have untold stress on the animals. Studies investigating the effect of stress on small ruminants' productivity have generally focused on a single stress at a time with limited information on the effect of multiple stresses on caprine pneumonia.

With the myriad of multiple stress factors that goats are constantly subjected to, there is need to understand the interactions between multiple stresses and diseases occurrence especially in different breeds of goats [7] and climatic conditions for proper planning for the control of respiratory diseases. Based on this, the observations of goats, subjected to repeated transport and dexamethasone treatments in the course of an experiment was hereby presented to highlight the effect of single and combination of stressors on the occurrence and severity of pneumonia especially in goats in a hot humid tropical environment.

\section{Materials and methods}

Animals: Sixteen, clinically healthy, Boer aged 2-3 years, non-pregnant does weighing between $23-25 \mathrm{~kg}$, were used and divided into four groups of four animals 
each. The animals were housed in well-ventilated sheds with asbestos roofing at $2.4 \mathrm{~m}$. The sheds were open from sides and the sheds were also maintained under proper hygienic conditions. The goats were kept in individual pens, fed on cut-grass and supplemented with commercial concentrates pellets. The goats were not immunised against any respiratory infections. Upon arrival to the experimental house, anthelmintic (Ivomectin, Suffolk, UK) was administered subcutaneously at the rate of $0.2 \mathrm{mg} / \mathrm{kg}$ body weight. The experiment started only when all goats were negative from carrying M. hemolytica and P. multocida.

Ethical approval: The experimental procedure was approved by the Animal Care and Use Committee of the Universiti Putra Malaysia. Drinking water was given ad libitum. Access to veterinary care was available at all time and the animal well-being was assessed regularly.

Experimental design: Group A were subjected to $8 \mathrm{~h}$ and $3 \mathrm{~h}$ transportation at four weeks interval, group B were subjected to $8 \mathrm{~h}$ transport stress once and four weeks later were given dexamethasone injection $\mathrm{i} / \mathrm{v}$ at $0.2 \mathrm{mg}$ per $\mathrm{kg}$ for 8 days. $8 \mathrm{~h}$ and $3 \mathrm{~h}$ transportation at four weeks, and the dexamethasone treatment was adopted to create additional stressful conditions that mimic a field situation. Group $C$ were subjected to $8 \mathrm{~h}$ transport only while Group D was not subjected to any stress. The All were housed separately in a standard goat farm pen, stocked at a rate of $1 \mathrm{~m}^{2} /$ goat [8]. All goats were observed clinically for respiratory signs while temperature was monitored daily and dead ones were necropsied while the survivors and all the goats in the control group were sacrificed on day 21 post transport or dexamethasone treatment.

The clinical signs were scored based on the modified scoring system as described by Gilmour et al. [9] as follows: demeanor (dull, depressed and recumbent) 1-3; nature of respiration (increased effort, labored, and dyspnea) 1-3; nasal discharge (mild, moderate, and profuse) 1-3; appetite (depressed and anorexic) 1-2; lacrimation (mild, moderate, and excessive) 1-3 and salivation (mild, moderate, and excessive) 1-3. A total clinical score was calculated for each animal in a group and the mean total clinical score for each group was subsequently calculated. The nasal swabs that were collected from all animals before and after day 7 post transport or dexamethasone treatment were subjected to cultural isolation.

Climatology and transportation: The experiment was carried out during April to July. The climate was hot and humid with average day temperature at $35^{\circ} \mathrm{C}$ and night temperature at $25^{\circ} \mathrm{C}$. The relative humidity fell within 70 to $90 \%$. The handling, loading and transportation of the experimental goats were carried out humanely in accordance with the guidelines governing animal transport welfare by road [10]. All the handling and loading procedures were conducted between 0900 and 1000 hours (GMT+1). A standard Bedford, open- roof pick-up van (Ford, UK), and popularly used in Malaysia for transportation of animals and goods, was used for the journey. The floor of the van was provided with beddings (wood shavings) covered with smooth sand for secure footing. The goats were stocked at a rate of $0.2 \mathrm{~m}^{2}$ per animal [11]. The journey commenced by 1000 hours and was terminated at 1800 hour for the first journey while the second journey was 3 hours. The vehicle travelled on a typical asphalt double lane road from Johor to Serdang $\left(3^{\circ} 00^{\prime} \mathrm{N}, 101^{\circ} 40^{\prime} \mathrm{E}\right)$ town and Serdang to Melaka, covering a total distance of about 350 and $140 \mathrm{~km}$ respectively. The vehicle travelled with an average speed of $50 \mathrm{~km} / \mathrm{h}$ and was driven on the first journey by two licensed drivers; each drove the vehicle for $4 \mathrm{~h}$ in a similar pattern. During the journey, the vehicle had two stop-overs and each stop-over time was less than $15 \mathrm{~min}$. During the resting period, the vehicle was parked in shade to avoid the direct effect of sunlight and rapid build-up of heat inside the vehicle. The goats were not fed or watered during the resting period [10].

\section{Bacterial isolation}

Detection of Mannheimia haemolytica and Pasteurella multocida: Nasal swab from each goat in each group post transport or dexamethasone treatment was smeared over half the surface of a seven per cent sheep blood agar plate prepared by pouring a thin layer of blood agar over a clear infusion base in order to aid till detection of characteristic haemolysis. Following aerobic incubation for $18 \mathrm{~h}$ at $37^{\circ} \mathrm{C}$, plates were examined for colonies resembling $M$. haemolytica, and P. multocida and representative colonies subcultured aerobically in $5 \mathrm{ml}$. of infusion broth for $18 \mathrm{~h}$ at $37^{\circ} \mathrm{C}$. These broth cultures of all the samples were cultured aseptically onto the agar (Oxoid, UK) and incubated at $37^{\circ} \mathrm{C}$ for 24 $\mathrm{h}$ for bacterial examination. Gram stain and biochemical tests were used to identify $M$. haemolytica, and $P$. multocida [12,13].

Pathology and immunohistochemistry: At day 21 posttransport or dexamethasone treatment, all surviving goats were euthanized in accordance with the Guidelines of the Animal Care and Use Committee, Universiti Putra Malaysia. During necropsy, attention was on the respiratory tracts. Presence of pleurisy was scored between 0 and 3;0 = no pleurisy and 3 being most severe. The gross lesions along the respiratory tract were described following visual examination and palpation before they were scored. Briefly, no significant lesions $=0,5-10 \%$ lung surface affected considered mild and scored 1 point, 11-25\% considered moderate and scored 2 points and over $25 \%$ considered severe and scored 3 points. [14] Representative tissue samples were collected from the nasal mucosa, trachea, and lungs and associated lymph nodes of the respiratory tract, liver, kidney and spleen were collected in $10 \%$ neutral buffered formalin for at least $12 \mathrm{~h}$. The samples were processed routinely for histopathology using the paraffin embedded technique, sectioned at 4 microns and stained with hematoxylin 

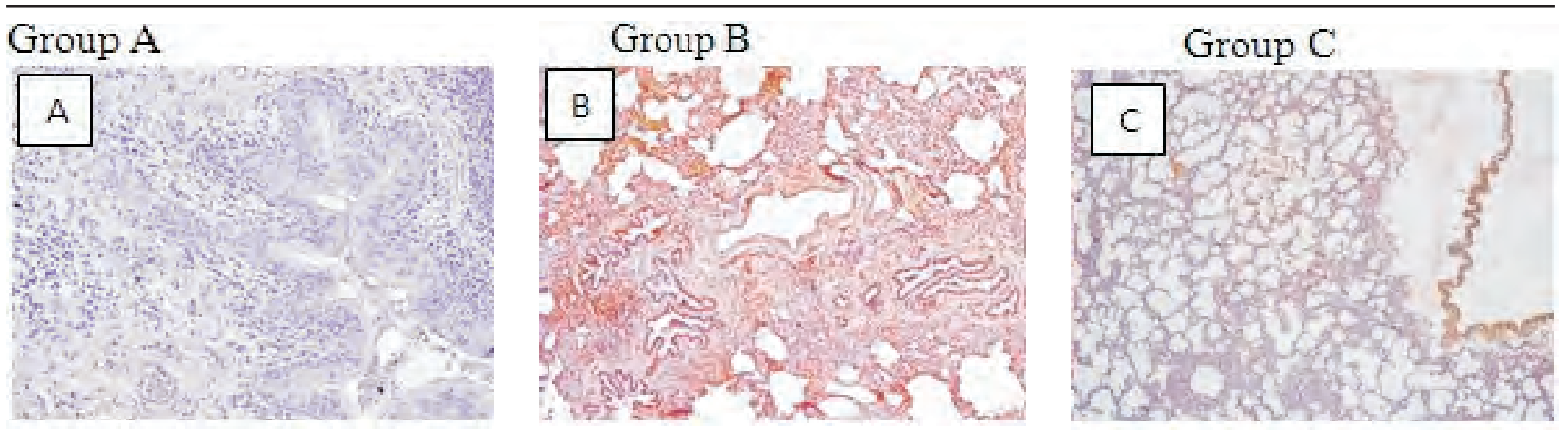

Figure-1. A: M. haemolytica antigens demonstrated by the red chromogen in the cytoplasm of alveolar macrophages, exudates in the bronchioles and in blood vessels B: in the cytoplasm of interstitial, alveolar macrophages, bronchial epithelium and in alveolar spaces and C: in the cytoplasm of alveolar, interstitial macrophages and pleura Avidin-biotin-peroxidase method
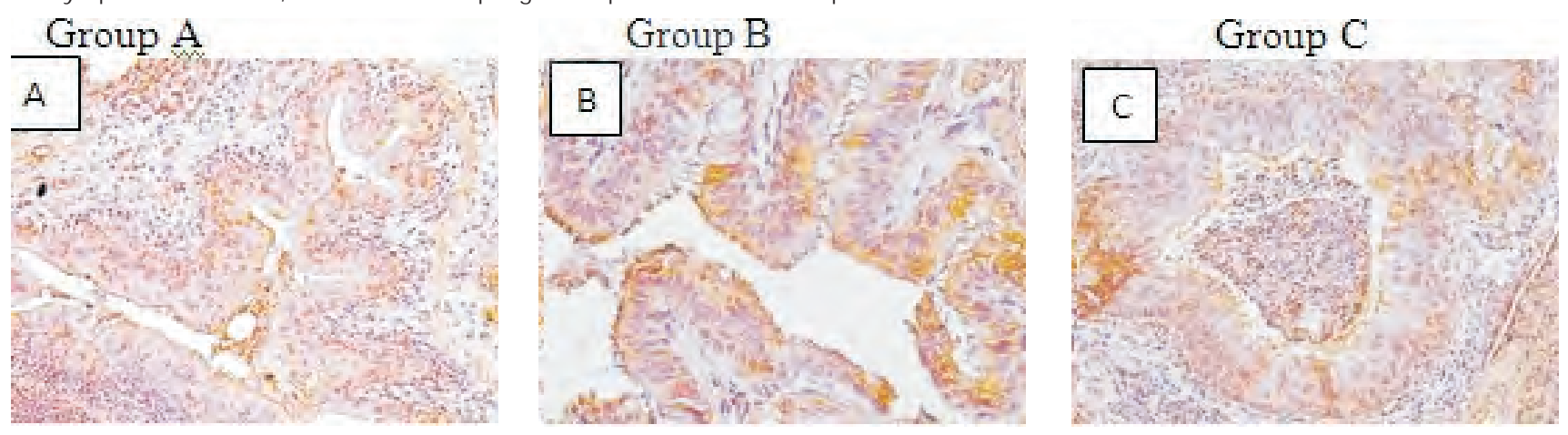

Figure-2. A. M. multocida antigens demonstrated by the red chromogen in the cytoplasm of alveolar macrophages, exudates around the bronchioles and in blood vessels B: in exudates in bronchioles and on the bronchiolar epithelium and $\mathbf{C}$ : in exudates in bronchioles and on the bronchiolar epithelium Avidin-biotin-peroxidase method

and eosin (H\&E). The histological lesions were recorded and overall grading for severity was assigned: No significant lesion $=0$; mild lesion $=1$; moderate lesion $=2$; and severe lesion $=3$.

Immunohistochemistry: The immunohistochemical detection of M. haemolytica, was as described by Yener et al., [15] while that of $P$. multocida was also based on the modification of Yener et al., [15]. The degree of immunostaining was assigned a score according to Yener et al., [15]. No significant immunostaining $=0$; slight immunostaining $=1$; moderate immunostaining $=2$; and deep immunostaining $=3$. Grading the staining on the nasal mucosa, bronchial and bronchiolar epithelial cells, pneumocytes, interstitial macrophages, pleura, in blood vessels and lymphatics. The representative pictures of the groups were presented in Figure-1 (M. haemolytica) and Figure-2 (P. multocida).

Statistical analysis: Mean total clinical signs, gross, histological lesions and immunohistochemical scores were summarized and subjected to Mann-Whitney test. All statistical procedures were estimated using the predictive analysis software 17 (PASW version 17; SPSS Inc., Chicago, IL, USA) and tested at $5 \%$ level of significance.

\section{Results}

Clinical observations: The clinical signs observed among goats of group A, B and C ranged between mild and moderate severity that included dullness, depression, anorexia, salivation, lacrimation, dyspnoea, coughing, nasal discharge and ear biting. The mean total clinical score was higher in group B than group A, however, two goats of the groups $\mathrm{A}, \mathrm{B}$ and $\mathrm{C}$ goats died 21,14 and 7 days post treatment period while the rest showed clinical signs of respiratory distress such as mild to moderate (Table-2).

Bacterial isolation: Isolation rate, the number of animal positive for the organism per evaluation time was more frequent from groups $\mathrm{A}$ and $\mathrm{B}$ than group $\mathrm{C}$ and $\mathrm{D}$. The isolation made from the lung was $100 \%$ (4/4) of group A and B, and 50\% (2/4) of group C. However, none of the group D had M. hemolytica $(\mathrm{MH})$ and P. multocida (PM) in the lungs and heart.

Pathology: As observed for clinical signs, the gross and histopathology lesions in surviving goats of groups A and $\mathrm{B}$ ranged between mild and moderate severity, which included acute rhinitis, acute fibrino-haemorrhagic bronchitis, acute fibrinous pleuropneumonia and pleurisy. (Table-2). Four goats (two from each group) that died in groups A and B showed severe hyperaemia and had mucoid discharge covering the nasal mucosa. Similar but less severe lesions were observed in goat that died on day 7 and sacrificed on day 21 in group C while lesions were absent from the remaining goats sacrificed in the groups including D.

The goats that died in each of the groups A, B and $\mathrm{C}$ had lung lesions typical of pneumonic pasteurellosis. An average of the lung area of dead animals were affected in repeated transport stressed group A 15\% (12-19\%), in group B $8.5 \%$ (2-16) and group C $6.5 \%$ (6-7) (Table-1) mostly involving the anterior and ventral parts of the lungs with more of the right lung. 
Table-1. Percentage pulmonary consolidation in goats subjected to single and combine dexamethasone treatment and successive road transport stress.

\begin{tabular}{|c|c|c|c|c|c|c|c|c|c|c|c|}
\hline Sr. No. & Groups & PD & $\begin{array}{c}\text { Left cranial } \\
5 \%\end{array}$ & $\begin{array}{c}\text { LPC } \\
6 \%\end{array}$ & $\begin{array}{c}\text { Left caudal } \\
32 \%\end{array}$ & $\begin{array}{c}\text { Accessory } \\
4 \%\end{array}$ & $\begin{array}{c}\text { Right cranial } \\
6 \%\end{array}$ & $\begin{array}{c}\text { RPC } \\
5 \%\end{array}$ & $\begin{array}{c}\text { Right middle } \\
7 \%\end{array}$ & $\begin{array}{c}\text { Right caudal } \\
35 \%\end{array}$ & $\begin{array}{l}\text { Total } \\
100 \%\end{array}$ \\
\hline 1. & A & 21D & 0 & 3.0 & 0 & 0.2 & 5.7 & 4.75 & 1.4 & 3.5 & 18.55 \\
\hline 2 & A & $21 D$ & 0 & 0.6 & 4.8 & 0 & 3.0 & 2.5 & 0.7 & 0 & 11.6 \\
\hline 3. & A & $21 S$ & 0 & 0 & 0 & 0 & 0 & 0 & 0 & 0 & 0 \\
\hline 4. & A & $21 S$ & 0 & 0 & 0 & 0 & 0 & 0 & 0 & 0 & 0 \\
\hline 5. & $B$ & 14D & 1.0 & 1.5 & 0 & 0 & 5.82 & 3.75 & 1.75 & 1.75 & 15.57 \\
\hline 6. & $\mathrm{~B}$ & $13 D$ & 0 & 0 & 0 & 0 & 1.5 & 0 & 0 & 0 & 1.5 \\
\hline 7. & B & $21 S$ & 0 & 0 & 0 & 0 & 0 & 0 & 0 & 0 & 0 \\
\hline 8. & $B$ & $21 S$ & 0 & 0 & 0 & 0 & 0 & 0 & 0 & 0 & 0 \\
\hline 9. & $\mathrm{C}$ & 7D & 0 & 3.6 & 1.6 & 0 & 0.54 & 0.7 & 0.21 & 0 & 6.65 \\
\hline 10. & $\mathrm{C}$ & $21 D$ & 0 & 0 & 0 & 0 & 4.5 & 1.25 & 0 & 0 & 5.75 \\
\hline 11. & $\mathrm{C}$ & $21 S$ & 0 & 0 & 0 & 0 & 0 & 0 & 0 & 0 & 0 \\
\hline 12. & C & $21 S$ & 0 & 0 & 0 & 0 & 0 & 0 & 0 & 0 & 0 \\
\hline 13. & D & $21 S$ & 0 & 0 & 0 & 0 & 0 & 0 & 0 & 0 & 0 \\
\hline 14. & D & $21 S$ & 0 & 0 & 0 & 0 & 0 & 0 & 0 & 0 & 0 \\
\hline 15. & D & $21 \mathrm{~S}$ & 0 & 0 & 0 & 0 & 0 & 0 & 0 & 0 & 0 \\
\hline 16. & D & $21 \mathrm{~S}$ & 0 & 0 & 0 & 0 & 0 & 0 & 0 & 0 & 0 \\
\hline
\end{tabular}

PD: Post-exposure days, LPC: Left Posterior Cranial, RPC: Right posterior cranial, D: Death, S: Sacrified

Table-2. The average mean survival time, clinical, lung consolidation, post mortem, histopathology and immunostaining scores for goats with different treatment groups.

\begin{tabular}{|c|c|c|c|c|c|c|c|c|}
\hline \multirow[t]{2}{*}{ Group } & \multirow[t]{2}{*}{ Treatment } & \multirow[t]{2}{*}{$\operatorname{MST}(n=4)$} & \multirow[t]{2}{*}{$\operatorname{cs}(n=4)$} & \multirow[t]{2}{*}{$\operatorname{LCS}(n=2)$} & \multirow[t]{2}{*}{ PMS ( $n=2)$} & \multirow[t]{2}{*}{$\operatorname{HPS}(n=2)$} & \multicolumn{2}{|c|}{ ISS ( $n=4)$} \\
\hline & & & & & & & MH & $\mathrm{PH}$ \\
\hline A & Successive Transport & 21 & $7.25 \pm 0.03^{*}$ & $15.1 \pm 4.0$ & $5.0 \pm 0.03^{*}$ & $6.0 \pm 0.02^{*}$ & $3.5 \pm 0.01^{*}$ & $9.0 \pm 0.01^{*}$ \\
\hline B & Transport+Dexa & 14 & $9.75 \pm 0.03^{*}$ & $8.4 \pm 7.17^{\star}$ & $5.5 \pm 0.02^{*}$ & $5.5 \pm 0.01^{*}$ & $6.0 \pm 0.02$ & $11.5 \pm 0.02 *$ \\
\hline $\mathrm{C}$ & Single transport & 14 & $3.25 \pm 0.02$ & $6.2 \pm 0.45^{*}$ & $2.5 \pm 0.01$ & $3.0 \pm 0.01$ & $4.0 \pm 0.03^{*}$ & $6.5 \pm 0.01$ \\
\hline D & Control & 0 & 0 & 0 & 0 & 0 & 0 & 0 \\
\hline
\end{tabular}

MST: Mean survival time, CS: Clinical score, LCS: Lung consolidation score, PMS: Postmortem score, HPS: Histopathology score, ISS: I mmuno-staining score, $\mathrm{MH}=$ Mannheimia haemolytica, $\mathrm{PH}=$ Pasteurella multocida, Values with the similar* are not significant

Affected parts appeared dark red and firm with distinct demarcation between the lesions and the normal lung areas. The remaining goats in groups A, B, C and D euthanized at day 21 showed no lung lesion.

Microscopic lesions: The main microscopic lesions observed in the nasal mucosa of goats in groups 1 include acute rhinitis, tracheitis, fibrino bronchitis, fibrinous pneumonia and pleura pneumonia.

Immunohistochemistry: The immunostaining was similar in terms of cells involved and spread while the intensity as graded showed that the immunostaining score was more in $\mathrm{PH}$ than $\mathrm{MH}$ and that of group B was more than $\mathrm{A}$ and $\mathrm{C}$ as shown in Table-2 where the pattern of the survival time, clinical, lung consolidation, postmortem, histopathology and immunostaining scores for goats with different treatment groups was summarised and presented.

\section{Discussion}

This investigation describes for the first time the effects of repeated transport on the occurrence and severity of pneumonia in goat. The effect of these treatments on the animal was evaluated using the clinical, gross, lung consolidation, histopathological and immunostaining score. Although the effect of transportation stress has been reported to affect many physiological parameters in goats $[16,17]$, the pathological derangements has not been accorded due attention.

This present study, further reaffirm the fact that dexamethasone in combination with other stress result in pneumonic pasteurellosis as earlier opined by Zamri-Saad et al., [4]. This study showed that repeated transport stress and combination of transport stress with other stressors as mimic with the use of dexamethasone influence the development of pneumonic pasteurellosis in goats. This showed the possible additive effects of repeated transport and dexamethasone treatment on transport stress induced caprine pneumonia when such goats are not challenged as earlier observed [4].

Although in this investigation, the goats were not infected, the possible stress induced immunosuppression [18] could have aided the proliferation of respiratory bacteria in the nasal cavity and subsequent invasion as earlier observed by Jasni et al., [19]. In this case, the enhancement of the fibrinous bronchopneumonia observed in dexamethasone treatment and repeated transport stress groups could have been associated with the level of immunosuppression. This proliferation and subsequent colonisation of $P$. multocida and M. haemolytica could have accounted for the significant lung consolidation, gross and histopathological changes observed in these groups when compared to the single transport stressed group. An average of $8.5 \%$ consolidation score and fifty percent of the animal affected in repeated transport stressed and dexamethasone treated group is comparable to $19 \%$ reported by Zamri-Saad et al., [4] in experimentally challenged stressed goats.

The findings of the immunostaining further corroborated that combined $P$. multocida and $M$. 
haemolytica infection occur naturally in goats [20] when stressed. The possible reason for the severe lesions observed in group A and B with intense immunostaining for $P$. multocida showed the emerging importance of $P$. multocida over $M$. haemolytica in the epidermiology of bacterial caprine pneumonia in Malaysia. This may be associated with the control measures put in place by farmers and the popular use of the M. haemolyica vaccine by farmers in Malaysia.

This investigation showed for the first time the immunohistochemical detection of P. multocida and $M$. haemolytica in Malaysian goats and this further reaffirm the importance of immunohistochemistry in some situations where bacteriological methods is difficult (antibiotic treatment, frozen material, autolytic material and others) [15]. It also eliminated many problems associated with other identification methods to detect respiratory bacterial antigens on fixed tissues [21].

The study proved the detrimental effects of repeated stresses on the severity of caprine pneumonia hence it is expedient to conclude that when two or more stressors occur simultaneously, the total impact may be severe caprine pneumonia which may lead to death.

\section{Conclusions}

Repeated stress results in severe caprine pneumonia. P. multocida seems to be more important in the epidemiology of stress induced bacterial caprine pneumonia in Malaysia

\section{Authors' contribution}

It was the post doctoral investigation of the first author. Other authors contributed equally. All authors read and approved the final manuscript.

\section{Acknowledgements}

This project was funded by the Ministry of Science, Technology and Innovation, Malaysia (MOSTI) under the Sciencefund 5450546. We would like to express our appreciation to all staff of post mortem and histopathology laboratory, Faculty of Veterinary Medicine, UPM for their excellent assistance.

\section{Competing interests}

Authors declare that they have no competing interest.

\section{References}

1. Mohamed, R. A., Abdelsalam E. B. (2008) A review on pneumonic pasteurellosis (respiratory Mannheimiosis) with emphasis on Pathogenesis, virulence mechanisms and predisposing factors, Bulg. J. Vet. Med, 11(3): 139-160.

2. Slocombe, R. F., Malark, J., Ingersoll, R., Derksen, F. J., Robinson, N. E. (1985) Importance of neutrophils in the pathogenesis of acute pneumonic pasteurellosis in calves, Am J Vet Res, 46: 2253-2258.

3. Averos, A., Martin, S., Riu, M., Serratosa, J., Gosalvez, L. F. (2008) Stress response of extensively reared young bulls being transported to growing-finishing farm under Spanish summer commercial conditions. Life Sci, 119: 174-182.

4. Zamri-Saad, M., Jasni, S., Nurida, A. B., Sheikh-Omar, A. R. (1991) Experimental Infection of Dexamethasone Treated
Goats with Pasteurella haemolytica A2, Br. Vet. J. 147 (6): 565-568.

5. Mohamed, R. A. (2002) The effect of iron compounds and other factors on the pathogenesis of pneumonic pasteurellosis in Nubian goats. 2002; Ph.D. Thesis, Faculty of Veterinary Science, University of Khartoum, Sudan.

6. Minka, N. S., Ayo, J. O., Sackey, A. K. B., Adelaiye, A. B. (2009) Assessment and scoring of stresses imposed on goats during handling, loading, road transportation and unloading, and the effect of pretreatment with ascorbic acid. Livest. Sci, 125: 275-282.

7. Sejian, V., Maurya, P., Kumar, K., Naqvi, S. M. K. (2012) Effect of Multiple Stresses (Thermal, Nutritional, and Walking Stress) on the Reproductive Performance of Malpura Ewes, Vet Med Inter, Article ID 471760, doi: $10.1155 / 2012 / 471760$.

8. Kannan, G., Terrill, T. H., Kouakou, B., Gelaye, S., Amoah, E. A. (2002) Simulated pre-slaughter holding and isolation effects on stress responses and live weight shrinkage in meat goats, JAnim Sci, 80: 1771-1780.

9. Glimour, N.J.L., Sharp, J. M., Glimour, J. S. (1982) Effect of oxytetracycline therapy on experimentally induced pneumonic pasteurellosis in lambs. Vet, Rec., 111:97-99.

10. Richardson, C. (2002). Lowering stress in transported goats. Ontario Ministry of Agriculture and Food-Livestock Technology Branch.

11. Idrus, Z., Bahyuddin, N., Wai, C. Y., Farjam, A. S., Sazili, A. Q., Rajion, M. A., Meng, G. Y. (2010) Physiological responses in goats subjected to road transportation under the hot, humid tropical conditions, Int. J. Agric. Biol, 12: 840844.

12. Lennetfe, E. H., Spaulding, E. H., Truant, J. P. (1974) Manual of Clinical Microbiology, p . 246. Washington: American Society for Microbiology.

13. Jablonski, P. E., Jaworski, M., Hovde, C. J., (1996) A minimal medium for growth of Pasteurella multocida, FEMS Microbiol Lett, 140:165-169.

14. Abubakar M. S and Zamri-Saad M. (2011) Clinicopathological changes in buffalo calves following oral exposure to Pasteurella multocida $\mathrm{B}: 2$, Basic and Applied Pathology, 4: 130-135 doi:10.1111/j.1755-9294.2011. 01113.x.

15. Yener, Z., Ilhan, F., Ilhan, Z., Saglam, Y. S. (2009) Immunohistochemical detection of Mannheimia (Pasteurella) haemolytica antigens in goats with natural pneumonia, Vet Res Commun, 33: 305-313.

16. Minka N. S and Ayo J. O. (2009) Physiological responses of food animals to road transportation stress Afr J Biotechnol, 8 (25): 7415-7427.

17. Rajion, M. A., Mohamed, I. S., Zulkifli, I., Goh Y. M. (2001) The effects of road transportation on some physiological stress measures in goats. Asian-Australian J. Anim. Sci., 14: $1250-1252$.

18. Chiang, Y. W., Roth, J. A., Andrews, J. J . (1990) Influence of recombinant bovine interferon gamma and dexamethasone on pneumonia attributable to Haemophilus somnus in calves, Am. J. Vet. Res, 51:759-762.

19. Jasni, S., Zamri-Saad, M., Mutalib, A. R., Sheikh-Omar, A. R. (1991) Isolation of Pasteurella haemolytica from the nasal cavity of goats, Br. Vet. J, 147(4):352-355.

20. Loganathan, P and Chandrasekaran, S. (1992) Clinicopathological changes in goats experimentally infected with Pasteurella multocida and Pasteurella haemolytica. In Proceedings of the National IRPA Seminar pp. 20-1. Ministry of Science, Technology and Environment, Malaysia.

21. Emikpe, B. O., Sabri, Y. M., Akpavie, S. O., Zamri Saad, M. (2010) Experimental infection of Peste des petits ruminants virus and Mannheimia haemolytica A2 in goats: Immunolocalisation of Mannheimia haemolytica antigens, Vet Res Commun, 34 (7): 569-578

$* * * * * * * *$ 